\title{
Are you certain about SIRT?
}

\author{
Friedrich C. Luft
}

Published online: 13 March 2014

(C) Springer-Verlag Berlin Heidelberg 2014

Histones, discovered by Albrecht Kossel in the late nineteenth century, are highly alkaline proteins found in cell nuclei that package and order DNA into structured units called nucleosomes. Histones are the protein components of chromatin. They act as spools around which the DNA is wrapped. Because of histones, $1.8 \mathrm{~m}$ of DNA in each mammalian cell nucleus can be packed into a nucleus about $4-8 \mu \mathrm{m}$ in diameter. Thus, the total $1.8-\mathrm{m}$ length is reduced to about $90 \mu \mathrm{m}$. This rate of packaging provides a length-to-width ratio of about 10 million to 1 . Histones are more than packaging tools. They are also the sites of gene regulation. Histones undergo posttranslational modifications, which greatly influences their interactions with DNA and other nuclear proteins. Existence of a protruding tail from the nucleosome allows histones to be covalently modified at predetermined sites. The mechanisms include methylation, acetylation, phosphorylation, ubiquitination, SUMOylation, citrullination, and ADPribosylation. Histone modifications are crucial to diverse biological processes such as gene regulation, DNA repair, and chromosome condensation (mitosis and meiosis). Histone deacetylases (HDACs) are a class of enzymes that remove acetyl groups $(\mathrm{O}=\mathrm{C}-\mathrm{CH} 3)$ from an $\varepsilon-N$-acetyl lysine amino acid on a histone. This removal from lysine allows the histones to wrap the DNA more tightly. As a result, DNA expression is regulated largely by acetylation and deacetylation. HDAC action is opposite to that of histone acetyltransferase activity. HDACs stem from an ancient protein superfamily, the sirtuins.

The sirtuins are an enzyme family that possesses ribosyltransferase, deacetylase, and similar enzymatic activities. The name comes from Silent Mating-type Information

F. C. Luft $(\bowtie)$

Experimental and Clinical Research Center, MDC/Charité,

Lindenbergerweg 80, 13125 Berlin, Germany

e-mail: luft@charite.de
Regulator, first discovered in yeast; sirtuins (SIRTs) are the human homologs. SIRT1 is an important member of the family that can function as a nicotinamide-adenine-nucleotide (NAD)-dependent HDAC. Thus, SIRT1 acts as an epigenetic gene that silences and suppresses recombination of DNA. SIRT1 itself can be downregulated. For instance, insulin resistance and insulin sensitivity influence SIRT1 regulation, which in turn acts to improve insulin sensitivity by repressing protein tyrosine phosphatase 1B [1]. A SIRT1 mutation was causative in a family of persons with type 1 diabetes [2]. A picture from SIRT1 research is emerging that places the enzyme at the hub of a complex molecular interaction array in which mostly nutrient-triggered signals are translated into adaptive cell responses [3]. The failure of SIRT1 function appears to underlie diseases as diverse as diabetes, neurodegenerative disorders, and even cancer. SIRT1 appears to connect moderate calorie intake to longevity, similar to eating only every other day. A high-fat diet triggers inflammation-induced cleavage of SIRT1 in adipose tissue [4]. A decline of SIRT1-related protective circuits over time may explain the effects of aging and overeating. As a matter of fact, the processes may be interrelated, connected together by SIRT1, or lack of SIRT1. Intimately tied to nutrition, SIRT1 turns up in discussions about cancer generation [5, 6]. SIRT1 is also implicated in Alzheimer's disease [7]. Can cardiovascular [8] and renal disease [9] lag far behind? SIRT1 seems to be approaching stardom as an enzyme for all occasions. Figure 1 displays an enthusiast's view of SIRT1 protective interactions. The message appears to be, "keep your DNA tightly wrapped."

Not surprisingly, the literature on SIRT1 and angiotensin (Ang) is extensive. We learn that heat-shock proteins ameliorate insulin resistance by augmenting Ang 1-7 signaling [10]. SIRT1 may act by epigenetically regulating angiotensin converting enzyme (ACE)-2 [11]. SIRT1 putatively modifies Ang IImediated endothelial cell dysfunction [12]. Indeed, SIRT1 appears to downregulate Ang II (AT1) receptors in vascular smooth 


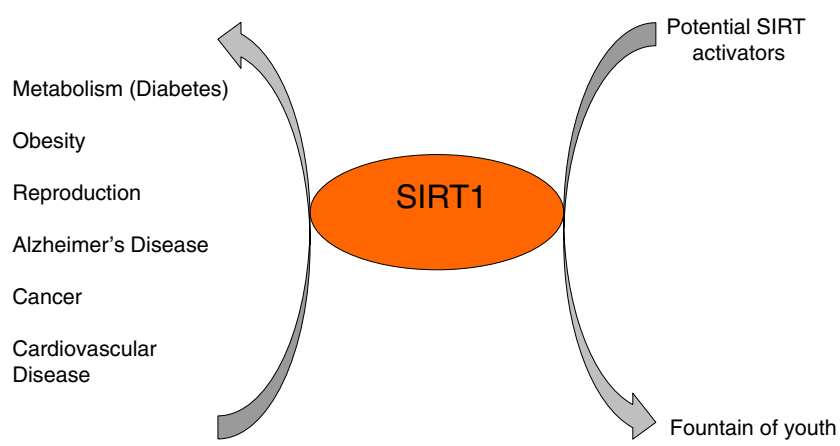

Fig. 1 One gene does all

muscle cells [13]. However, this issue of $J$ Mol Med carries the SIRT1-Ang II story further. Gao and colleagues overexpressed SIRT1 in vascular smooth muscle cells (VSMCs) to test the actions of Ang II [14]. In initial experiments, they found that Ang II infusion decreased the expression and activity of SIRT1 in mouse aortas. The investigators had at their disposal a crucial model to test the next hypothesis, a VSMC-specific SIRT1 transgenic mouse [15]. This SIRT1-overexpressing mouse exhibited less blood pressure elevation with Ang II infusion. Furthermore, SIRT1 overexpression alleviated vascular remodeling in mouse thoracic and renal aortas induced by Ang II infusion, and decreased reactive oxygen species (ROS) generation, vascular inflammation, and collagen synthesis in arterial walls. Transforming growth factor- $\beta 1$ (TGF- $\beta 1$ ) was decreased, as was Ang II-induced nuclear factor- $\mathrm{kB}$ activation. The authors conclude that SIRT1 overexpression in VSMCs reduces blood pressure and inhibits Ang II-induced vascular remodeling.

Sounds great, you say? How can I become a SIRT1 transgenic mouse? The question is not farfetched since small molecular activators of SIRT1 are being developed [16]. SIRT1-activating compounds (STAC) appear to provide health benefits in animal disease models. Recent data point to a common mechanism of allosteric activation by natural and synthetic STAC involving STAC binding to a conserved $\mathrm{N}$-terminal domain in SIRT1. These compounds are not to be confused with polyphenols such as resveratrol. A STAC would have been an interesting control in the current report [14]. Distinctly missing from this report and from a similar paper from this group is how Ang II influences SIRT1 expression on a molecular level [17]. Numerous models come to mind to study this issue. However, this basic bit of molecular biology is absent and could perhaps answer the question, whether or not all the salubrious actions of SIRT1 in Fig. 1 are really true.

Respectfully,

Friedrich C. Luft

\section{References}

1. Sun C, Zhang F, Ge X, Yan T, Chen X, Shi X, Zhai Q (2007) Sirt1 improves insulin sensitivity under insulin-resistant conditions by repressing ptp1b. Cell Metab 6:307-319

2. Biason-Lauber A, Boni-Schnetzler M, Hubbard BP, Bouzakri K, Brunner A, Cavelti-Weder C, Keller C, Meyer-Boni M, Meier DT, Brorsson C et al (2013) Identification of a sirt1 mutation in a family with type 1 diabetes. Cell Metab 17:448455

3. Fusco S, Maulucci G, Pani G (2012) Sirt1: def-eating senescence? Cell Cycle 11:4135-4146

4. Chalkiadaki A, Guarente L (2012) High-fat diet triggers inflammation-induced cleavage of sirt1 in adipose tissue to promote metabolic dysfunction. Cell Metab 16:180-188

5. Lin Z, Fang D (2013) The roles of sirt1 in cancer. Genes Cancer 4: 97-104

6. Wang Z, Chen W (2013) Emerging roles of sirt1 in cancer drug resistance. Genes Cancer 4:82-90

7. Braidy N, Jayasena T, Poljak A, Sachdev PS (2012) Sirtuins in cognitive ageing and Alzheimer's disease. Curr Opin Psychiatry 25: 226-230

8. Corbi G, Bianco A, Turchiarelli V, Cellurale M, Fatica F, Daniele A, Mazzarella G, Ferrara N (2013) Potential mechanisms linking atherosclerosis and increased cardiovascular risk in COPD: focus on sirtuins. Int J Mol Sci 14:12696-12713

9. Kitada M, Kume S, Koya D (2014) Role of sirtuins in kidney disease. Curr Opin Nephrol Hypertens 23:75-79

10. Karpe PA, Tikoo K (2013) Heat shock prevents insulin resistance induced vascular complications by augmenting angiotensin-(1-7) signalling. Diabetes Nov 22. [Epub ahead of print]

11. Clarke NE, Belyaev ND, Lambert DW, Turner AJ (2014) Epigenetic regulation of angiotensin-converting enzyme 2 (ace2) by sirt1 under conditions of cell energy stress. Clin Sci 126:507-516

12. Marampon F, Gravina GL, Scarsella L, Festuccia C, Lovat F, Ciccarelli C, Zani BM, Polidoro L, Grassi D, Desideri G et al (2013) Angiotensin-converting-enzyme inhibition counteracts angiotensin ii-mediated endothelial cell dysfunction by modulating the p38/sirt1 axis. J Hypertens 31:1972-1983

13. Miyazaki R, Ichiki T, Hashimoto T, Inanaga K, Imayama I, Sadoshima J, Sunagawa K (2008) Sirt1, a longevity gene, downregulates angiotensin ii type 1 receptor expression in vascular smooth muscle cells. Arterioscler thromb Vasc Biol 28:12631269

14. Gao P, Xu TT, Lu J, Li L, Xu J, Hao DL, Chen HZ, Liu DP (2013) Overexpression of sirt1 in vascular smooth muscle cells attenuates angiotensin ii-induced vascular remodeling and hypertension in mice. J Mol Med. doi 10.1007/s00109-013-1111-4

15. Li L, Zhang HN, Chen HZ, Gao P, Zhu LH, Li HL, Lv X, Zhang QJ, Zhang R, Wang Z et al (2011) Sirt1 acts as a modulator of neointima formation following vascular injury in mice. Circ Res 108:1180 1189

16. Sinclair DA, Guarente L (2014) Small-molecule allosteric activators of sirtuins. Annu Rev Pharmacol Toxicol 54:363-380

17. Li L, Gao P, Zhang H, Chen H, Zheng W, Lv X, Xu T, Wei Y, Liu D, Liang C (2011) Sirt1 inhibits angiotensin ii-induced vascular smooth muscle cell hypertrophy. Acta Biochim Biophys Sin 43: 103-109 